$$
y_{0}^{2 m}
$$


Distribuclón del ingreso e incidencia de la pobreza a lo largo del ajuste

Oscar Altimir

Nuevas orientaciones para la gestión pública

Eugenio Lahera

Industrias petroquímica y de máquinas herramientas:

estrategias empresariales

Daniel Chudnovsky, Andrés López y Fernando Porta

Productividad, crecimiento y exportaciones industriales de Brasil

Regis Bonelli

Maquila en el Caribe: la experiencia de Jamaica

Larry Willmore

Elasticidad-precio de las exportaciones agrícolas de Centroamérica

De la inflaclón crónica a la inflación moderada en el Ecuador

Luis I. Jácome Hidalgo

Nuevas estrategias de las empresas transnacionales en la Argentina

Bernardo Kosacoff y Gabriel Bezchinsky

Informalidad y pobreza en América Latina

Guillermo Rosenbluth

Crisis y alternativas en los procesos de regionalización

Sergio Boisier

Una perspectiva cultural de las propuestas de la CEPAL

Fernando Calderón, Martín Hopenhayn y Ernesto Ottone

La CEPAL y el neoliberalismo: entrevista a Fernando Fajnzylber

Orientaciones para los colaboradores de la Revista de la CEPAL

Publicaciones reclentes de la CEPAL 


\section{Elasticidad - precio de las exportaciones agrícolas de Centroamérica}

\section{Alberto Gabriele \\ Oficial de Asuntos Económi- cos, Sede Subregional de la CEPAL en México.}

Las economías de los países centroamericanos (Costa Rica, El Salvador, Guatemala, Honduras y Nicaragua) son muy dependientes de cuatro productos de exportación tradicionales: el banano, el café, el algodón y el azúcar. Sólo a fines de los años ochenta comenzó a disminuir el porcentaje de las exportaciones de Centroamérica que éstos representan, que sigue siendo de alrededor de un 50\%. En este artículo se analizan los factores determinantes de las tendencias de la producción de esos cuatro productos, reconociendo la importancia de los factores económicos y sociales no relacionados con los precios y de las perturbaciones naturales y las provocadas por el ser humano. Se ha utilizado la base de datos de la Sede Subregional de la CEPAL en México para estimar la elasticidad-precio de la oferta de los cuatro productos durante el período 1960-1990 y se han puesto a prueba modelos simplificados de regresión lineal en los que sólo se consideran los precios como variables explicativas. En los casos del banano y el azúcar no se encontró ninguna relación estadística significativa entre la producción y el precio. Al parecer, únicamente la producción de café reacciona en cierta medida a las variaciones de precios de un año a otro. Sólo se pudo calcular las elasticidades de la oferta del café, que son bastante bajas. Se concluyo que no se debería sobrestimar el potencial de expansión de las exportaciones de los países pobres y pequeños mediante la aplicación de políticas ortodoxas que influyan sobre todo en los precios macroeconómicos. 


\section{El rol de los productos agrícolas de exportación tradicional en las economías de Centroamérica}

Los países de Centroamérica (Costa Rica, El Salvador, Guatemala, Honduras y Nicaragua) siguen siendo muy dependientes de las exportaciones agrícolas tradicionales. El banano, el café, el algodón y el azúcar representaron en promedio el $57 \%$ de las exportaciones de toda la región en el decenio de 1980.

CUADRO 1

Centroamérica: Participación relativa de los cuatro productos agrícolas de exportación tradicional en las exportaciones totales

(Porcentajes)

\begin{tabular}{|c|c|c|c|c|}
\hline & $1970-1979$ & 1980-1989 & 1990 & 1991 \\
\hline \multicolumn{5}{|l|}{ Banano } \\
\hline Costa Rica & 23 & 23 & 23 & 26 \\
\hline El Salvador ${ }^{a}$ & $\ldots$ & $\ldots$ & $\cdots$ & $\ldots$ \\
\hline Guatemala & 3 & 6 & 7 & 7 \\
\hline Honduras & 29 & 33 & 45 & 43 \\
\hline Nicaragua & 1 & 5 & 8 & 11 \\
\hline Centroamérica & 9 & 15 & 19 & 19 \\
\hline \multicolumn{5}{|l|}{ Café } \\
\hline Costa Rica & 31 & 28 & 18 & 18 \\
\hline El Salvador & 45 & 59 & 45 & 37 \\
\hline Guatemala & 36 & 34 & 27 & 23 \\
\hline Honduras & 25 & 24 & 22 & 20 \\
\hline Nicaragua & 22 & 34 & 21 & 14 \\
\hline Centroamérica & 33 & 35 & 25 & 22 \\
\hline \multicolumn{5}{|l|}{ Algodón } \\
\hline Costa Rica ${ }^{a}$ & $\ldots$ & $\ldots$ & $\ldots$ & $\ldots$ \\
\hline El Salvador & 9 & 4 & $\ldots$ & $\ldots$ \\
\hline Guatemala & 13 & 6 & 2 & 2 \\
\hline Honduras ${ }^{a}$ & 1 & 1 & $\ldots$ & $\ldots$ \\
\hline Nicaragua & 24 & 21 & 11 & 18 \\
\hline Centroamérica & 9 & 4 & $l$ & 1 \\
\hline Azúcar & & & & \\
\hline Costa Rica & 4 & 2 & 2 & 2 \\
\hline El Salvador & 4 & 3 & 3 & 6 \\
\hline Guatemala & 8 & 6 & 13 & 12 \\
\hline Honduras & 1 & 3 & 1 & 2 \\
\hline Nicaragua & 5 & 5 & 12 & 12 \\
\hline Centroamérica & 5 & 4 & 6 & 6 \\
\hline \multicolumn{5}{|c|}{$\begin{array}{l}\text { Productos agrícolas de } \\
\text { exportación tradicional }\end{array}$} \\
\hline Costa Rica & 56 & 52 & 43 & 45 \\
\hline El Salvador & 58 & 66 & 48 & 44 \\
\hline Guatemala & 59 & 52 & 50 & 44 \\
\hline Honduras & 55 & 61 & 69 & 64 \\
\hline Nicaragua & 53 & 65 & 52 & 55 \\
\hline Centroamérica & 57 & 57 & 51 & 49 \\
\hline
\end{tabular}

Fuente: CEPAL, Sede Subregional de la CEPAL en México, 1992b, cuadro 30.

a Los tres puntos (...) indican una participación relativa inferior a $1 \%$.

El autor agradece los comentarios y la ayuda de varios colegas y desea agradecer en particular a Huberth Escaith por su valioso asesoramiento en el campo de la econometría y a Juan Pérez Ga- briel por su paciente colaboración en el área de la computación. Por supuesto, no se puede responsabilizar a ninguno de ellos por los errores y deficiencias que puedan subsistir en el texto. 
En ningún país fueron inferiores al 50\%, y en Honduras y Nicaragua superaron el $60 \%$. El peso relativo de los cuatro productos sólo comenzó a disminuir paulatinamente a fines de los años ochenta y en 1991 fue inferior al 50\% en toda la región por primera vez (cuadro 1).

Los productos de exportación tradicionales siguen teniendo una importancia fundamental en las economías centroamericanas, pese a que no se han mantenido al margen de la crisis económica general que ha venido afectando a la región desde comienzos de los años ochenta, lo que se debe tanto a las tendencias desfavorables de los precios internacionales (salvo para el banano) como a la falta de progreso técnico en el proceso productivo. Durante el período comprendido entre 1980 y 1989 el valor en dólares de las exportaciones centroamericanas se redujo en promedio a una tasa anual de $1.8 \%$, mientras que el de los cuatro principales productos de exportación tradicionales disminuyó a una tasa anual de 2.3\% (CEPAL, Sede Subregional de la CEPAL en México, 1992b, cuadro 30.) La crisis tuvo efectos muy marcados en el caso del algodón, que prácticamente dejó de ser una fuente de divisas, en tanto que las exportaciones de banano fueron las únicas cuyo valor aumentó a lo largo de la década, a una tasa media de $4.4 \%$. La producción de algodón se redujo en forma dramática, a una tasa anual media de $12.8 \%$, y la de los demás productos sólo registró un incremento marginal (café, $0.8 \%$; banano, $1.2 \%$

CUADRO 2

Centroamérica: Tasas de crecimiento de la producción y las exportaciones de banano, café, algodón y azúcar

(Porcentajes)

\begin{tabular}{|c|c|c|c|c|c|c|c|c|}
\hline & & Produc & & & & Export & iones & \\
\hline & $1970-1979$ & $1980-1989$ & 1990 & 1991 & $1970-1979$ & $1980-1989$ & 1990 & 1991 \\
\hline Banano & & & & & & & & \\
\hline $\begin{array}{l}\text { Costa Rica } \\
\text { El Salvador }\end{array}$ & 2.1 & 3.5 & 8.1 & 5.6 & 12.3 & 3.7 & 13.9 & 20.7 \\
\hline Guatemala & 3.3 & -1.2 & 4.0 & 1.7 & 3.9 & 7.5 & -1.0 & -7.1 \\
\hline Honduras & 1.8 & -0.2 & -4.2 & -7.0 & 12.1 & 4.0 & 4.2 & -9.1 \\
\hline Nicaragua & 20.5 & -2.1 & 7.4 & 8.9 & 41.0 & 9.6 & 34.5 & 5.9 \\
\hline Centroamérica & 2.5 & 1.2 & 3.2 & 1.1 & 11.9 & 4.4 & 8.1 & 3.5 \\
\hline Café & & & & & & & & \\
\hline $\begin{array}{l}\text { Costa Rica } \\
\text { El Salvador } \\
\text { Guatemala } \\
\text { Honduras } \\
\text { Nicaragua }\end{array}$ & $\begin{array}{l}2.3 \\
3.7 \\
3.0 \\
8.9 \\
4.0\end{array}$ & $\begin{array}{r}3.6 \\
-1.5 \\
1.0 \\
3.9 \\
-2.9\end{array}$ & \begin{tabular}{r|r}
13.1 \\
-7.6 \\
4.8 \\
14.9 \\
-35.5
\end{tabular} & $\begin{array}{r}-6.1 \\
-1.6 \\
-2.5 \\
-11.5 \\
45.6\end{array}$ & $\begin{array}{l}17.6 \\
22.2 \\
17.6 \\
25.2 \\
19.5\end{array}$ & $\begin{array}{r}1.6 \\
-9.3 \\
-2.2 \\
-0.9 \\
1.0\end{array}$ & $\begin{array}{r}-14.3 \\
1.6 \\
-16.8 \\
-5.2 \\
-20.8\end{array}$ & $\begin{array}{r}8.5 \\
-13.5 \\
-11.1 \\
-14.3 \\
-48.1\end{array}$ \\
\hline Centroamérica $^{b}$ & 3.8 & 0.8 & $\cdots$ & -2.7 & 20.1 & -3.8 & -10.8 & -10.2 \\
\hline Algodón & & & & & & & & \\
\hline $\begin{array}{l}\text { Costa Ricab } \\
\text { El Salvador } \\
\text { Guatemala } \\
\text { Honduras } \\
\text { Nicaragua }\end{array}$ & \begin{tabular}{r|r}
16.7 \\
2.4 \\
11.3 \\
17.7 \\
-14.1
\end{tabular} & \begin{tabular}{r|}
20.4 \\
-19.9 \\
-11.8 \\
-15.6 \\
-11.7
\end{tabular} & $\begin{array}{r}-24.1 \\
-8.0 \\
-1.5 \\
20.6\end{array}$ & $\begin{array}{r}-50.9 \\
-15.0 \\
8.8 \\
-64.7 \\
-5.7\end{array}$ & $\begin{array}{r}2.5 \\
15.9 \\
24.6 \\
29.7 \\
16.5\end{array}$ & $\begin{array}{r}-40.4 \\
-18.8 \\
-25.1 \\
1.0\end{array}$ & $\begin{array}{r}62.5 \\
-9.7 \\
\ldots \\
12.9\end{array}$ & $\begin{array}{r}23.1 \\
-12.2 \\
\ldots \\
28.9\end{array}$ \\
\hline Centroamérica & 2.6 & -12.8 & 0.5 & -0.3 & 19.6 & -15.9 & 1.7 & 12.7 \\
\hline Azúcar & & & & & & & & \\
\hline $\begin{array}{l}\text { Costa Rica } \\
\text { El Salvador } \\
\text { Guatemala } \\
\text { Honduras } \\
\text { Nicaragua }\end{array}$ & $\begin{array}{r}2.3 \\
8.6 \\
10.0 \\
7.1 \\
1.4\end{array}$ & $\begin{array}{r}-1.5 \\
0.6 \\
5.4 \\
-0.8 \\
-0.8\end{array}$ & $\begin{array}{r}11.1 \\
21.9 \\
12.5 \\
8.8 \\
16.8\end{array}$ & $\begin{array}{r}7.9 \\
13.5 \\
4.7 \\
8.1 \\
-3.4\end{array}$ & $\begin{array}{r}17.1 \\
16.1 \\
21.6 \\
30.6 \\
8.0\end{array}$ & $\begin{array}{r}-10.3 \\
1.3 \\
3.2 \\
-10.6 \\
4.0\end{array}$ & $\begin{array}{l}69.9 \\
34.4 \\
66.0 \\
11.8 \\
31.6\end{array}$ & $\begin{array}{r}17.3 \\
72.9 \\
-7.6 \\
22.8 \\
-19.0\end{array}$ \\
\hline Centroamérica & 6.1 & 1.8 & 13.7 & 5.9 & 17.2 & -0.7 & 53.8 & 1.2 \\
\hline
\end{tabular}

Fuente: CEPAL, Sede Subregional de la CEPAL en México, $1992 \mathrm{~b}$, cuadros 21 y 30.

a Las tasas de crecimiento de las exportaciones se calculan de acuerdo con su valor en dólares corrientes.

b Los tres puntos (...) indican que las tasas de crecimiento no son aplicables debido a que los niveles de producción y de exportación son nulos o despreciables. 
y caña de azúcar, 1.8\%). En Nicaragua disminuyó la producción de los cuatro cultivos y sólo en Guate- mala se registró un considerable incremento del volumen general de producción ${ }^{1}$ (cuadro 2).

\section{II}

\section{Importancia de los factores ajenos a los precios}

La Sede Subregional de la CEPAL en México realizó recientemente un estudio sobre la situación y las perspectivas de las exportaciones agrícolas tradicionales en Centroamérica (CEPAL, Sede Subregional en México, 1992b.) Uno de los temas centrales del estudio es la evolución de la producción y de los principales factores que influyeron en ella.

Dicha evolución se debió a diversos factores económicos y no económicos, muchos de los cuales no son cuantificables y ni siquiera pueden compararse entre sí. Los conflictos armados provocaron prolongados trastornos en las zonas rurales de El Salvador y Nicaragua y, en menor medida, de Guatemala. Las condiciones climáticas afectaron a la producción de distintas maneras, que no se reflejan en los datos pluviométricos ni en la incompleta información histórica sobre los frecuentes desastres naturales.

También incidieron en las tendencias de la producción varios otros factores sociales y económicos no relacionados con los precios. La capacidad de los agricultores para realizar todas las actividades necesarias durante el ciclo productivo y para invertir con el objeto de ampliar su capacidad productiva, así como su disposición a hacerlo, dependieron más de la disponibilidad efectiva de créditos y de los controles impuestos a su utilización que de la tasa de interés. El valor de mercado y la liquidez de los productos agrícolas de exportación y, por lo tanto, los incentivos para producirlos, se vieron limitados por reformas inadecuadas de los mecanismos comerciales, fiscales y del mercado, como también por obstáculos legales, físicos y de infraestructura que afectaron al transporte y al comercio internos. La disponibilidad de mano de obra y la oferta de productos básicos manufacturados disminuyeron drásticamente en muchas zonas rurales debido a los conflictos armados, lo que redujo el poder real de compra de los saldos monetarios de los agricultores $\mathrm{y}$, por consiguiente, los incentivos a la producción. ${ }^{2}$ En algunos países la incertidumbre con respecto a los derechos de propiedad contribuyó a desestimular la inversión en nuevas instalaciones productivas e incluso en el mantenimiento de las instalaciones existentes.

Los trastornos ambientales provocados por el ser humano, cuyo alcance es difícil calcular con precisión, también influyeron en la situación descrita; el más importante de ellos fue la reducción de la fertilidad a largo plazo provocada por el uso excesivo de pesticidas y otros productos químicos en el cultivo del algodón.

\section{III}

\section{Modelo simplificado}

Pese a la reconocida importancia de los factores ajenos a los precios, se recurrió a la información sobre el período 1960-1990 almacenada en la base de datos de la Sede Subregional de la CEPAL en México, en un intento por poner a prueba la hipótesis muy general de que existe una correlación
1 La información proviene de la base de datos de la CEPAL y de estimaciones preliminares de la producción de 1991, basadas en cifras oficiales. En CEPAL, Sede Subregional de la CEPAL en México (1992b), se presenta más información estadística sobre las tendencias de la producción y de las exportaciones agrícolas tradicionales en Centroamérica.

\footnotetext{
2 Por ejemplo, en muchas áreas rurales de Nicaragua el racionamiento de productos básicos, la desestabilización del comercio debido al conflicto y la hiperinflación, que se prolongaron durante gran parte del último decenio, limitaron dramáticamente los incentivos de los pequeños agricultores para incrementar sus ingresos.
} 
positiva entre los precios al productor y los niveles de producción.

Desde fines de los años cuarenta se han venido realizando estudios sobre la sensibilidad al precio en el sector agrícola, especialmente en lo que se refiere al comportamiento de los pequeños agricultores y en particular en el continente africano. Por ejemplo, Maitha (1969) y Ady (1968) determinaron que la oferta de café presentaba elasticidades positivas aunque moderadas en Kenya y Uganda, respectivamente; Olayide (1972) llegó a una conclusión similar con respecto al algodón en Nigeria. Los resultados de éstos y varios otros estudios -en muchos de los cuales no se pudieron establecer correlaciones significativas entre producción y precioshan sido comentados por Helleiner (1975), quien determinó que, si bien contribuyen a refutar el mito de que los pequeños agricultores suelen tener un comportamiento "irracional" o "no económico", las conclusiones también muestran las limitaciones de tomar exclusivamente en consideración los precios al productor, así como la complejidad de los patrones de adopción de decisiones de los agricultores. En los últimos años ha continuado el debate teórico y de política, alimentado por interpretaciones contradictorias de nuevos hallazgos empíricos. Balassa (1990), Berg (1989) y Bond (1983), entre otros, han hecho hincapié en la importancia de la respuesta a los precios de la oferta de los agricultores, incluso en contextos tradicionales. Por otra parte, Helleiner, Lele, Lipton y Mellor se cuentan entre los autores que se oponen a que se otorgue excesiva importancia a las políticas de precios, junto con mostrarse partidarios de la adopción de estrategias agrícolas globales y a largo plazo destinadas a estimular las exportaciones (véanse, entre otros, Gbetibouo, Lele y Van de Walle, 1989; Lipton, 1987, y Mellor 1990). Aparentemente, gran parte de la información generada en los años ochenta confirmó las opiniones de estos últimos, como lo reconoce, por ejemplo, el Banco Mundial (1990).

\footnotetext{
3 Como se sabe, la validez del supuesto de la maximización de las utilidades está limitada por diversos factores, entre los cuales cabe mencionar los siguientes: el hecho de que la información sea con frecuencia incompleta y asimétrica, lo que contribuye al surgimiento de mecanismos de creación de expectativas complejos y heterogéneos en las diferentes categorías de productores; la posibilidad de que los pequeños agricultores muestren una cierta inclinación a la producción de subsistencia, lo que puede conducir a que se valorice la mano de obra familiar en cero o en un nivel inferior al valor de mercado, y la preferencia de los terratenientes adinerados por los cultivos tradicionales, que se explica por motivos culturales y de prestigio.
}

En los estudios sobre los productos de exportación tradicionales de Centroamérica, al igual que en otros estudios similares, se debería haber incluido en el modelo utilizado el costo de los insumos y, en los casos pertinentes, el precio de los cultivos que competían con los productos considerados, dado que, según el fundamento a nivel microeconómico de la sensibilidad al precio, los agricultores que se dedican a cultivos de exportación maximizan sus utilidades cuando existen obstáculos a la producción. ${ }^{3}$ Fue imposible tomar en cuenta el costo de los insumos, debido a la limitada cobertura de los datos disponibles, que no incluyen series completas de los precios de los insumos y de los productos alternativos que podrían competir por el uso de la tierra con los cuatro productos agrícolas de exportación tradicional. ${ }^{4}$ De hecho, la base de datos no contiene un índice agregado del precio de los granos, que pueden competir con el algodón por el uso de tierras bajas irrigadas; sin embargo, en los intentos preliminares por utilizar los precios de los granos como variables explicativas en los modelos correspondientes al algodón quedó demostrado que la influencia que éstos ejercen es del signo correcto pero no es estadísticamente significativa. En cuanto al precio de los insumos, los limitados datos disponibles para los años ochenta reflejan una marcada tendencia ascendente (cuadro 3), que sin duda contribuyó en gran medida a desestimular la producción, especialmente en el caso del algodón, el cultivo que exige un mayor volumen de insumos de los cuatro cultivos tradicionales considerados.

En vista de que los datos disponibles sólo proporcionaban información parcial, decidimos poner a prueba modelos de regresión lineal muy simplificados, en los que sólo se utilizan como variables explicativas los precios de los productos, cuya influencia en la producción puede verse modificada en algunos casos por variaciones del tipo de cambio o disturbios populares.

\footnotetext{
4 Esto es lo que ocurre, por ejemplo, con el precio del ganado, que junto con las expectativas sobre la existencia de mercados en los que se pueda colocar las exportaciones puede ser un importante factor determinante de las decisiones de inversión de los productores de café.
} 
CUADRO 3

Centroamérica: Indice de costos de insumos en cuatro países centroamericanos ${ }^{a}$

\begin{tabular}{|c|c|c|c|c|c|c|c|c|c|c|c|}
\hline & 1980 & 1981 & 1982 & 1983 & 1984 & 1985 & 1986 & 1987 & 1988 & 1989 & 1990 \\
\hline \multicolumn{12}{|l|}{ Costa Rica } \\
\hline Fertilizantes & $\ldots$ & $\ldots$ & 100 & 84 & 107 & 129 & 124 & 122 & 133 & 133 & 138 \\
\hline Herbicidas & $\ldots$ & $\ldots$ & 100 & 77 & 120 & 138 & 158 & 374 & 157 & 172 & 195 \\
\hline Insecticidas & $\ldots$ & $\ldots$ & 100 & 91 & 105 & 131 & 143 & 145 & 176 & 195 & 216 \\
\hline \multicolumn{12}{|l|}{ El Salvador } \\
\hline Fertilizantes & 100 & 121 & 130 & 103 & 121 & 105 & 183 & 171 & 161 & 169 & 184 \\
\hline \multicolumn{12}{|l|}{ Guatemala } \\
\hline Fertilizantes & $\ldots$ & $\cdots$ & $\cdots$ & $\ldots$ & 100 & 122 & 149 & 168 & 188 & 195 & 202 \\
\hline Pesticidas & $\ldots$ & $\ldots$ & $\ldots$ & $\ldots$ & 100 & 122 & 150 & 177 & 272 & 255 & 238 \\
\hline \multicolumn{12}{|l|}{ Honduras } \\
\hline Insecticidas & 100 & 115 & 132 & 146 & 144 & 148 & 149 & 149 & 157 & 151 & 271 \\
\hline Herbicidas & 100 & 136 & 133 & 139 & 135 & 127 & 129 & 124 & 127 & 171 & 286 \\
\hline Fertilizantes & 100 & 119 & 121 & 122 & 107 & 101 & 99 & 91 & 92 & 115 & 190 \\
\hline
\end{tabular}

Fuente: CEPAL, Sede Subregional de la CEPAL en México, 1992b, cuadro 22.

a Los índices de precios de las categorías de productos incluidas en el cuadro son promedios simples de los índices correspondientes al precio de un solo producto.

\section{IV}

\section{Diferencias estructurales entre los cuatro productos de exportación tradicional}

Los resultados del estudio coincidieron con los que se preveía obtener. Quedó demostrado que era imposible establecer correlaciones econométricas estadísticamente significativas entre los precios y la producción de banano y azúcar en todos los países sobre la base de modelos relativamente sencillos. Era previsible que el estudio arrojara este resultado en el caso de estos dos productos, dado que sus precios nominales en los mercados nacionales e internacionales son indicadores absolutamente inadecuados de rentabilidad.

Como se sabe, la rentabilidad del comercio del banano y las decisiones relativas a su producción dependen fundamentalmente de las estrategias de un número reducido de empresas transnacionales, que controlan los mercados mundiales de este producto, desde la etapa de cultivo hasta las redes de distribu- ción en los países industrializados. ${ }^{5}$ Es lógico suponer que, en un contexto competitivo de carácter colusorio u oligopólico, las empresas determinan los precios internacionales de acuerdo con su percepción de las tendencias de la demanda en los principales mercados, en tanto que, desde el punto de vista de la empresa, los precios a nivel de la explotación agrícola no pasan de ser precios contables internos. Las empresas transnacionales también determinan los precios al productor y las metas de producción de las pequeñas plantaciones. Por otra parte, en contados

\footnotetext{
5 Además, en la última década ha habido un muy limitado equilibrio oligopólico, debido a las frecuentes absorciones de empresas y las también frecuentes entradas y salidas de empresas del mercado, todo lo cual ha contribuido a la inestabilidad de la relación entre producción y precios.
} 
casos los productores de algunos países latinoamericanos han establecido canales independientes de comercialización, pero como siguen controlando una porción muy reducida de los mercados mundiales, no tienen influencia en los precios. Aunque en menor medida que en el caso de otros productos de exportación tradicionales, las políticas discriminatorias de importación y comercio del banano que aplican los países industrializados también contribuyen a complicar la relación entre los precios vigentes en Europa, los Estados Unidos y otras regiones desarrolladas, por una parte, y las expectativas y el comportamiento de los productores de Centroamérica, América del Sur, Africa y Asia, por otra. ${ }^{6}$

Este último fenómeno es mucho más marcado en el caso del azúcar. La intervención de los gobiernos en los principales países desarrollados fragmenta y distorsiona los mercados mundiales; dichos gobiernos no sólo otorgan una gran protección a sus agricultores y refinadores, sino que además hacen distinciones unilaterales entre los exportadores de azúcar de los países en desarrollo, mediante la aplicación de cuotas y la fijación de precios arbitrarios de compra. ${ }^{7}$

Además, debido a las características técnicas de las industrias del banano y el azúcar, los niveles de producción anual dependen en gran medida de las cuantiosas inversiones en infraestructura realizadas en el pasado con propósitos muy específicos y que responden a estrategias de largo plazo. ${ }^{8}$ Este es otro factor de rigidez, que dificulta aún más el estudio econométrico de la relación entre precios y producción.

Por otra parte, en los casos del café y el algodón no existe una integración vertical entre las actividades de producción y comercialización, al menos en Centroamérica. Aunque los grandes terratenientes han desempeñado tradicionalmente un papel de fundamental importancia, un alto porcentaje de la producción proviene de medianos y pequeños agricultores, especialmente en el caso del café. Además, incluso los grandes productores de café y algodón son centroamericanos en su mayoría, y actúan como agentes que no influyen en los precios cuando venden en los mercados mundiales, controlados por empresas transnacionales de comercialización con sede en países desarrollados. ${ }^{9}$

En el estudio se obtuvieron resultados estadísticamente significativos con respecto al café en la mayoría de los países, y en Guatemala y El Salvador con respecto al algodón; estos resultados concuerdan con lo que se sabía previamente sobre las características de la producción y comercialización de ambos productos en Centroamérica.

\section{V}

\section{Análisis empírico ${ }^{10}$}

\section{Café}

En principio, los precios deberían incidir en la producción de café debido a su influencia en las decisiones relativas a la siembra y a la renovación, en un ciclo de cinco a seis años, y en la poda, la fertilización y otras actividades anuales. ${ }^{11} \mathrm{De}$ hecho, no se encontró ningu-

6 Véanse, entre otros, Ellis (1984); López (1986); Naciones Unidas, Consejo Económico y Social (1989); Martínez Cuenca (1991); OCDE (1991), y UNCTAD (1992).

7 Véanse, entre otros, UNCTAD (1990 y 1992); CEPAL, Sede Subregional de la CEPAL en México (1992a); Viatte y Cahill (1991), y Davenport (1988).

8 Véanse, entre otros, Brown (1987); López (1986), y Naciones

Unidas, Consejo Económico y Social (1989).

9 Véase, por ejemplo, UNCTAD (1992).

10 En esta sección sólo se presentan los principales resultados del análisis.

11 Al término del ciclo anual de producción, todas las decisiones na relación estadísticamente significativa entre la producción y los precios cuando había un desfase de cinco a seis años entre ambos. Este resultado negativo no sólo podría indicar que los datos son inadecuados, sino también que las grandes inversiones en los cafetales no dependen del precio del grano, sino de otros factores económicos y posiblemente sociales. 
En cambio, cuando se utilizaron como variables explicativas los precios con un desfase de un año en regresiones logarítmicas en las que también se incluyeron uno o dos factores endógenos desfasados, los resultados fueron satisfactorios en todos los países con la excepción de Nicaragua. En Costa Rica, Guatemala y Honduras se utilizaron como variables explicativas los precios internos (al productor) en dólares, ${ }^{12}$ en tanto que en El Salvador el empleo del precio mundial y del tipo de cambio como variables explicativas independientes permitió un ajuste óptimo. ${ }^{13} \mathrm{El}$ coeficiente del tipo de cambio presentó el signo previsto (negativo), lo que indica en principio que las devaluaciones influyeron positivamente en la producción de café.

En el cuadro 4 se presentan las elasticidades a corto y largo plazo de la producción de café con respecto al precio y, en el caso de El Salvador, también con respecto al tipo de cambio. Las elasticidades a corto plazo son bajas, lo que sugiere que las medidas de política destinadas a elevar el precio al productor en términos reales con el objeto de incrementar la producción (por ejemplo, mediante subsidios, bajos impuestos netos, tipos de cambio múltiples e incluso una devaluación general) tendrían efectos positivos pero limitados. ${ }^{14}$ Las elasticidades a largo plazo se presentan sobre todo con el propósito formal de ofrecer datos estadísticos completos, pero tienen escaso valor informativo, debido a que sólo se puede demostrar la existencia de una correlación positiva a corto plazo entre la producción y el precio.

Centroamérica (cuatro paises): Elasticidades de la producción de café con respecto a los precios ${ }^{\mathrm{a}}$ y los tipos de cambio

\begin{tabular}{|c|c|c|c|c|}
\hline & \multicolumn{2}{|c|}{ Precio } & \multicolumn{2}{|c|}{ Tipo de cambio } \\
\hline & Corto plazo & Largo plazo & Corto plazo & Largo plazo \\
\hline El Salvador & 0.1386 & 0.2014 & -0.1908 & -0.2886 \\
\hline Guatemala & 0.1513 & 0.3721 & & \\
\hline Costa Rica & 0.0779 & 0.7853 & & \\
\hline Honduras & 0.1931 & 0.8915 & & \\
\hline
\end{tabular}

${ }^{a}$ Las elasticidades de precios corresponden al precio al productor nacional expresado en dólares en los casos de Costa Rica, Guatemala y Honduras, y al precio internacional en el de El Salvador.

\section{Algodón}

En el caso de este producto sólo se obtuvieron resultados aceptables para El Salvador y Guatemala, mediante el uso de modelos dinámicos simples en los que se realiza una regresión de la tasa de crecimiento de la producción a corto plazo con respecto a la

12 Según el supuesto implícito, los productores calculan su poder adquisitivo real sobre la base del valor en dólares de sus ingresos, en lugar de considerar el volumen de entradas en moneda nacional. Esto puede responder a su interés en adquirir bienes de capital o de consumo y servicios que sólo pueden comprarse en divisas (dentro o fuera del país) o a su costumbre de prever las consecuencias inflacionarias de las devaluaciones.

13 Sin embargo, esta regresión presenta un $R^{2}$ bajo (0.633). También se puso a prueba una variable ficticia (GUERRA) para el período 1980-1990. Estas regresiones no dieron resultados adecuados y el coeficiente de regresión y el coeficiente $t$ de la variable ficticia fueron extremadamente bajos. Este resultado negativo permite suponer que la guerra no afectó mayormente la producción de café en los años ochenta. En cambio, la variable ficticia GUERRA resultó ser significativa en el caso de la producción de algodón. tasa de incremento de los precios, también a corto plazo.

De hecho, en primer término se realizaron regresiones dinámicas generales en las que se indica el nivel de producción al lado izquierdo y los valores desfasados de la producción y de los precios a la derecha, como en el caso del café; los resultados apuntan a una especificación en primera diferencia. Por lo tanto, se modificó el modelo con el objeto de estimar la correlación entre las tasas de crecimiento de las variables.

14 Por supuesto, esta observación es válida siempre que las condiciones del mercado sean "normales" en términos generales. En la situación actual, en que los precios internacionales del café son tan bajos que algunos productores están sufriendo pérdidas netas, cabe considerar que los subsidios gubernamentales son necesarios para evitar el derrumbe de todo el sector. 
Se utilizó el siguiente modelo original con un año de desfase:

$P=f\left(P_{t-1}, p_{t}, p_{t-1}\right)$

Esto también podría expresarse mediante la siguiente ecuación logarítima-lineal:

(1) $\log (P)=C+a \log \left(P_{t-1}\right)+b_{1} \log \left(p_{t}\right)+b_{2} \log$ $\left(p_{t-1}\right)$,

siendo:

$\mathrm{P}=$ producción

$\mathrm{p}=$ precio

Incorporando la restricción $\mathrm{b}_{1}=-\mathrm{b}_{2}=\mathrm{b}$ y restando el $\log \left(\mathrm{P}_{t-1}\right)$ de ambos miembros, se obtiene el siguiente modelo simplificado:

(2) $\log (P)-\log \left(P_{t-1}\right)=C+(a-1) \log \left(P_{t-1}\right)+b$ $\left[\log \left(p_{t}\right)-\log \left(p_{t-1}\right)\right]$,

ó

(3) $\dot{P}=C+(a-1) \log \left(P_{t-1}\right)+\dot{b p}$

siendo:

$\dot{\mathrm{p}} \mathrm{y} \dot{\mathrm{p}}$ tasas de crecimiento

Se puede hacer el cálculo correspondiente a la ecuación (2) para Guatemala y El Salvador y agregarle la variable ficticia GUERRA en el último caso (con un valor 1 en el período 1980-1990 y 0 en los demás años). Los coeficientes $t$ de la variable de precios VARPRINAL y las estadísticas de prueba son satisfactorios, aunque el estadígrafo $R^{2}$ es inferior (poco más de 0.5 en Guatemala y menos de 0.4 en El Salvador), lo que indica que los precios ejercen escasa influencia en la evolución de la producción.

Los coeficientes de la variable VARPRINAL $\left(\log \left(p_{t}\right)-\log \left(p_{t-1}\right)\right)$, que pueden interpretarse como elasticidades a corto plazo de las tasas de crecimiento de la producción con respecto a las tasas de crecimiento de los precios al productor son 0.4635 en El Salvador y 0.7556 en Guatemala, y podrían indicar que existe una marcada correlación positiva a corto plazo entre las variaciones de los precios y las decisiones de siembra.

Es posible que el comportamiento de los productores responda al hecho de que, a diferencia de los cafetales, las tierras adecuadas para el cultivo del algodón son bajas y también sirven para otros cultivos, por ejemplo de granos. Todos los años los productores deben decidir si van a cultivar algodón (opción que exige cuantiosas inversiones a corto plazo) $\mathrm{u}$ otra especie. Por lo tanto, la variación del precio del algodón de un año a otro puede influir notablemente en la producción a corto plazo. ${ }^{15}$

El coeficiente de regresión y el coeficiente $\mathrm{t}$ de la variable endógena desfasada LPROAR (-1), que se podrían considerar como un reflejo de la influencia de factores de largo plazo en las variaciones de la producción, fueron altos y significativos en el caso de Guatemala, y bajos y no significativos en el de El Salvador. Para comprobar la hipótesis de que los factores económicos de largo plazo no relacionados con los precios al productor del algodón tienen efectos muy distintos tanto en Guatemala como en El Salvador, se realizaron regresiones de control para ambos países en las que no se consideró la variable LPROAR (-1). En comparación con el caso base, el ajuste de regresión se redujo drásticamente en el caso de Guatemala, pero sólo marginalmente en el de El Salvador. Estos resultados podrían indicar que la falta de estabilidad política en El Salvador contribuyó a limitar el comportamiento de los productores a respuestas de muy corto plazo, en tanto que los productores guatemaltecos tuvieron la oportunidad de desarrollar mejor las estrategias de producción, al menos en cierta medida.

Por último, cabe señalar que en El Salvador la variable ficticia GUERRA es significativa y tiene un alto coeficiente negativo $(-0.33)$, lo que refleja el efecto desestabilizador que tuvo el conflicto armado para la producción de algodón.

\footnotetext{
15 La experiencia práctica de los productores confirma esta explicación. Dicha experiencia fue comentada por Alfredo Gil Spillari, de la Junta Nacional del Algodón de Guatemala, en el Seminario sobre la situación de los productos agrícolas tradicionales de exportación, que se realizó en la Sede Subregional de la CEPAL en México (Ciudad de México, 10 y 11 de diciembre de 1992). Este argumento no niega la existencia de una correlación positiva a largo plazo entre la producción y los precios, que podría reflejarse en los coeficientes de la variable endógena desfasada y en la capacidad explicativa de las regresiones, además de la influencia de otras variables que inciden en la evolución de la producción. De hecho, aunque el valor de $\mathrm{R}^{2}$ es aceptable, resulta inferior a 0.8 en el caso de Guatemala. En El Salvador es superior, pero los valores que arrojan las pruebas de Durbin y de Ramsey son altos y sólo parcialmente aceptables, lo que permite pensar que puede haber tanto una autocorrelación como problemas de mala especificación.
} 


\section{VI}

\section{Resumen y conclusiones}

El ejercicio descrito demuestra que la respuesta de la producción de productos agrícolas de exportación tradicional de Centroamérica a los estímulos provenientes de los precios no es muy importante; en algunos casos no fue posible confirmarla estadísticamente.

Los resultados del ejercicio indican que se debe proceder con cautela en la determinación de las ventajas de los programas de ajuste y liberalización que se están llevando a cabo en países pequeños y pobres con una estructura tradicional, rígida y no diversifica-

16 En varios estudios recientes se ha llegado a conclusiones similares. Véanse, entre otros, Daniel, Green y Lipton (1985); Aziz (1990); Lipton (1987); Mullor-Sebastian (1990); Taylor (1988), y Banco Mundial (1990). Véase también Brinkman y Gabriele (1992). Los resultados de un cálculo econométrico basado en datos correspondien- da de exportaciones, dado que no cabe esperar que las variaciones de la producción derivadas del mercado sean de gran alcance, por lo menos en el corto y mediano plazo. ${ }^{16} \mathrm{El}$ débil efecto de los precios sugiere que es importante complementar las medidas de ajuste y propiciar la modernización y la diversificación, por ejemplo mediante políticas industriales específicas destinadas a encauzar la mayor parte de las importaciones adicionales a sectores reconocidamente aptos para incrementar las exportaciones.

(Traducido del inglés)

tes al decenio de 1980 demuestran que, sobre todo en el caso de Costa Rica, las exportaciones en general no presentan elasticidad con respecto a los precios y que, por consiguiente, los subsidios a las exportaciones no constituyen una política adecuada (Hoffmeister, 1991, p. 19).

\section{Bibliografía}

Ady, Peter (1968): Supply functions in tropical agriculture, Bulletin of the Oxford University Institute of Economic and Statistics, vol. $30, \mathrm{~N}^{\circ} 2$.

Aziz, Sartaj (1990): Agricultural Policies for the 1990s, Paris, Organización de Cooperación y Desarrollo Económicos (OCDE).

Balassa, Bela (1990): Incentive policies and export performance in Sub-Saharan Africa, Structural Change in African Agriculture, IFPRI Policy Briefs, No 5, Washington, D.C., Instituto Internacional de Investigaciones sobre Políticas Alimentarias.

Banco Mundial (1990): Making Adjustment Work for the Poor. A Framework for Policy Reform in Africa. Washington, D.C.

Berg, Elliot (1989): The liberalisation of rice marketing in Madagascar, World Development, vol. 17, $\mathrm{N}^{\circ} 5$, Nueva York, Pergamon Press plc. mayo.

Bond, Marian E. (1983): Agricultural responses to prices in SubSaharan African countries, Staff Papers, vol. $30, \mathrm{~N}^{\circ} 4$, Washington, D.C. Fondo Monetario Internacional (FMI), diciembre.

Brinkman, Henk-Jan y Gabriele Alberto (1992): Problems in Agricultural Development in Sub-Saharan Africa, Working Paper, $N^{\circ} 17$, Nueva York, Naciones Unidas, Departamento de Asuntos Económicos y Sociales Internacionales (DAESI).

Brown, James J. (1987): The International Sugar Industry - De velopments and Prospects, World Bank Staff Commodity Working Papers, $\mathrm{N}^{\circ}$ 18, Washington, D.C., Banco Mundial.

CEPAL, Sede Subregional de la CEPAL en México (1992a): Las negociaciones sobre liberalización agrícola en la Ronda Uruguay del GATT. Perspectivas de Cetroaméroca, México, D.F.

(1992b): Centroamérica: productos tradicionales de exportación. Situación y perspectivas, LC/MEX/R. 373 (SEM. 52/2), México, D.F.

Daniel, Philip, Reginal Green y Michael Lipton (1985): A strategy for the rural poor, Journal of Development Planning, $\mathrm{N}^{\circ} 15$,
Nueva York, Naciones Unidas. Publicación de las Naciones Unidas. $\mathrm{N}^{\circ}$ de venta E.85.II.A.6.

Davenport, Michael (1988): European Community Trade Barriers to Tropical Agricultural Products, O.D.I. Working Paper, $\mathrm{N}^{\circ}$ 27, Londres, Overseas Development Institute (ODI).

Ellis, Frank (1984): Las transnacionales del banano en Centroamérica, San José.

Gbetibouo, Mathurin, Uma Lele y Nicolás van de Walle (1989): Cotton in Africa, An Anlysis of Differences in Performance, MADIA Discussion Paper, $N^{\circ} 7$, Washington, D.C.

Helleiner, Gerald (1975): Smallholder decision making: tropical African evidence, Lloyd G. Reynolds (ed.). Agriculture in Development Theory, Londres.

Hoffmeister, Alexander (1991): The Cost of Export Subsidies: Evidence from Costa Rica, IMF Working Paper (WP/91/94), Washington, D.C., FMI.

Lipton, Michael (1987): Limits of price policy for agriculture: Which way for the World Bank, Development Policy Review, vol. 5, $\mathrm{N}^{\circ} 2$, Londres, ODI.

López, José R. (1986): La economía del banano en Centroaméri$c a$, San José.

Maitha, J.K. (1969): A supply function for Kenian coffee, Eastern African Economic Review, vol. $1 . \mathrm{N}^{\circ} 1$.

Martínez Cuenca, Alvaro (1991): Banana libre, Managua.

Mellor, John W. (1990): Emphasizing agriculture in economic development -Is it a risky business?, Structural Change in African Agriculture, IFPRI Policy Briefs, $\mathrm{N}^{\circ} 5$, Washington, D.C.

Mullor-Sebastian, Alicia (1990): Export Instability and Policy Implications for Developing Countries as Residual Suppliers, IMF Working Paper (WP/90/44), Washington, D.C., FMI.

Naciones Unidas, Consejo Económico y Social (1989): Trends in the International Market for Agricultural and Tropical Products and the Liberalization of International Agricultural Trade, Report of the Secretary General, Nueva York. 
OCDE (1991): Agricultural Policies, Markets and Trade, Monitoring and Outlook 1991, París.

Olayide Olajuwon (1972): Some estimates of supply elasticities for Nigeria's cash crops, Journal of Agrcultural Economics, vol. $23, \mathrm{~N}^{\circ} 3$.

Taylor, Lance (1988): Varieties of Stabilization Experiences: Towards Sensible Macroeconomics in the Third World, Oxford, Clarendon Press.
UNCTAD (1990): The Implications of Bilateral Arrangements on Trade and Development of Developing Countries, Informe de la Secretaria, Ginebra.

(1992): Analytical report by the UNCTAD Secretariat at the Conference, UNCTAD VII, Ginebra.

Viatte, Gérad y Carmel Cahill (1991): The resistance to agricultural reform, The OECD Observer, $\mathrm{N}^{\circ} 171$, París, OCDE, agosto-septiembre. 